\author{
Fredrik Falkenström, ${ }^{1}$ Matthew David Gee, ${ }^{2}$ Mary Wangari Kuria, ${ }^{3}$ \\ Caleb Joseph Othieno ${ }^{4}$ and Manasi Kumar ${ }^{5}$
}

\begin{abstract}
'Senior Lecturer and Researcher, Centre for Psychotherapy Research and Education, Centre for Psychiatry Research, Department of Clinical Neuroscience, Karolinska Institutet, and Stockholm Health Care Services, Stockholm County Council, Sweden
Care Services, Stocking

2 Specialty Registrar in Medical Psychotherapy Oxford Health NHS Foundation Trust, Warneford Hospital, Oxford, UK; Honorary Teaching Fellow, Department of Psychiatry, University of Oxford, UK

${ }^{3}$ Chairman and Associate Professor, Department of Psychiatry, College of Health Sciences, University of Nairobi Kenya

${ }^{4}$ Associate Professor, Department of Psychiatry, College of Health Sciences, University of Nairobi, Kenya

${ }^{5}$ Senior Lecturer, Department of Psychiatry, College of Health Sciences, University of Nairobi, Kenya; Honorary Research Fellow, Research Department of Clinical Health and Educationa Collonge sychology, University College London, UK; email manni_3in@ hotmail.com
\end{abstract}

This paper is the first in a planned series of papers studying the effectiveness of psychotherapy and counselling in Nairobi. It describes a method for checking the effectiveness of psychotherapy and improving service quality in a Kenyan context. Rather than prematurely imposing psychotherapy protocols developed in Western countries in another cultural context, we believe that first studying psychological interventions as they are practised may generate understanding of which psychological problems are common, what interventions therapists use, and what seems to be effective in reducing psychiatric problems. The initial step is to assess outcome of psychological treatments as they are conducted. This is followed by statistical analyses aimed at identifying patient groups who are not improving at acceptable rates. Therapists will then be trained in a 'best practice' approach, and controlled trials are used in a final step, testing new interventions specifically targeted at patient groups with sub-optimal outcomes.

Pragmatic ways of delivering psychotherapy and counselling in public hospitals in low- and middleincome countries (LMICs), such as task sharing and task shifting, have improved treatment delivery (Chowdhary et al, 2014), but the evidence base for these non-manualised interventions is thin. Lack of evidence, however, does not necessarily imply lack of effectiveness. This paper describes a method for checking effectiveness and improving service quality in a Kenyan context. Rather than prematurely imposing psychotherapy protocols developed in Western countries in another cultural context, we believe that first studying psychological interventions as they are practised may generate understanding of which psychological problems are common, what interventions therapists use, and what seems to be effective in reducing psychiatric problems in a LMIC.

\section{Psychotherapy and counselling in Nairobi, Kenya}

The use of psychotherapy and counselling in Nairobi's public hospitals is growing. Kenyatta National Hospital's centre to support patients diagnosed with HIV and medical problems has expanded to provide psychosocial interventions to a wide range of patients, reflecting expanding liaison psychiatry services in other African tertiary hospitals too, for example in Nigeria (Issa et al, 2015). Challenges include few qualified mental health nurses, with even fewer employed in public hospitals. Therapeutic models in Kenya are often eclectic, influenced by Western psychological models, pastoral counselling in churches and the HIV counselling programme. Sociocultural and economic factors, including availability of training, acceptability of appointments and time boundaries, transport availability and treatment cost, modify patients' and therapists' behaviours to create a contextualised, pragmatic style of therapy varying from Western treatment protocols. Studies that have evaluated psychological interventions in Kenya have examined culturally adapted cognitive-behavioural therapy to reduce alcohol use among HIV-infected patients (Papas et al, 2011), psychoeducation for depression, suicidality, anxiety and substance misuse (Muriungi \& Ndetei, 2013) and motivational interviewing to change pregnant women's health-seeking behaviours (Gisore et al, 2014). These, however, may not reflect psychological interventions as they are actually practised.

\section{How do we improve outcomes?}

Psychotherapy research has made significant progress in the past three decades and a number of treatments have been empirically supported (Roth \& Fonagy, 2005). However, the field is divided as to which research design is most suitable for the study of the effects of psychotherapy. The currently most influential research has taken the blueprint from medical research, using the randomised controlled trial (RCT) as the gold standard. However, although strong for establishing causality, many researchers have noted that the RCT is often limited in external validity, that is, in generalisability to the patients, therapists and settings of usual care (Falkenström, 2010). In the settings of LMICs, implementation of these treatments and whether these are evidence based have now become areas of great debate (Murray et al, 2014). However, the conditions within which treatments do or do not work have received very little research attention, but are crucial for effective practice. A treatment may work well under the more or less optimal conditions of an RCT study but not in regular practice, or vice versa.

An alternative (albeit complementary) research design that has gained momentum in 
psychotherapy research focuses on the process and outcome of treatments as they are conducted in routine practice: a strategy termed practice-based evidence (e.g. Holmqvist et al, 2015). In addition to enhancing ecological validity, research findings may be easier to communicate to practitioners since data are more closely related to actual practice than findings from laboratory conditions. The dominant strategy for linking academic psychotherapy research to clinical practice so far has been dissemination of RCT findings to practising therapists. In the extreme, this strategy may be viewed as 'empirical imperialism' (Lampropoulos et al, 2002): researchers imposing on practitioners their views on what practitioners should do regardless of the needs and opinions of practitioners and patients.

It is likely that some therapists and clinics are already achieving good results with their patients, or at least with some groups of patients. It would be a disservice to change their practice on the basis of evidence established in other settings that may not be applicable. Additionally, dissemination without regard to patient and clinician preferences is likely to be unsuccessful. Meeting patient preferences with regard to psychotherapy orientation leads to better outcomes (Swift et al, 2013). Similarly, clinicians have different talents for, and commitment to, various types of psychotherapy, and this affects outcome. The risk of blindly disseminating treatments 'borrowed' from elsewhere without understanding system barriers or competencies is that well functioning clinics are forced to change their ways of working, and that the new methods fit less well with clinician aptitudes and patient preferences, resulting in reduced quality of care. The risk of the harmful effects of disseminating evidence-based therapies developed in Western cultures to non-Western countries without regard to local organisational and sociocultural conditions is probably even higher, since therapists are likely to have adapted their methods to local cultural conditions.

\section{Combining practice-based and RCT methodology}

Our proposed strategy uses practice-based research as a starting point for 'mapping the territory' before conducting clinical trials. Instead of starting with the researchers' ideas of which treatments should be tested, we propose the use of a four-step inductive strategy:

- first, data are gathered from routine clinical practice to find out which groups of patients are not being helped by current practice

- once enough data have been collected the second step is to analyse the data in order to identify subgroups of patients who are not improving at expected rates

- after identifying subgroups of patients with inferior outcomes, the researchers identify the treatment(s) with the best research evidence for the identified patient subgroups, and therapists will then be trained in this 'best practice' approach

- an RCT is then conducted to test whether this treatment is superior to treatment as usual with these particular patient subgroups.

The proposed strategy thus combines practicebased and RCT methodologies, which will likely result in better use of resources.

\section{Improving the effectiveness of psychological treatments in Nairobi}

Using this strategy, we have collected data on patients treated with psychological interventions in Nairobi's public hospitals. Data have been collected at Mathari Hospital - a specialist psychiatric referral hospital - and the out-patient psychiatric clinic, mental health department and youth clinic of Kenyatta National Hospital - a 1500-bed national teaching and referral hospital. The latter two departments offer psychiatric treatment, psychological therapy, social work and counselling to adults and adolescents respectively. Any patient receiving an intervention described by practitioners as 'counselling' or 'psychotherapy' was included after giving written informed consent. The data will soon be analysed to identify patient groups who have suboptimal outcomes. In addition, qualitative interviews are being conducted with patients and therapists in order to explore cultural beliefs, expectations, and attitudes to mental health treatments. Information from these interviews will be important in outlining culturally adapted treatments for mental health problems.

\section{Preliminary findings}

There seems to be an over-representation of men among patients seeking therapy in Nairobi, as they made up about two-thirds of the sample of 241 participants. This is an unusual finding that differs from most data from Western countries, where men display less help-seeking behaviour for psychological problems than women (MöllerLeimkühler, 2002). In addition, more than half the patients attended only one session, with numbers roughly halving for each subsequent session. Only around $5-10 \%$ of patients attended more than three sessions. Most evidence for psychological therapies worldwide is based on attendance over at least 10-12 sessions. Our patients are not receiving anything approaching this, highlighting the need to research contextualised therapy in this setting. Patients have informally shared that they discontinue therapy early because of difficulties mainly with finances, long and difficult journeys to attend appointments or mismatch with regular medical appointments. It may also be that there is disharmony in the working alliance or disarray in addressing treatment preferences with the patients, resulting in increased attrition, issues that we are exploring in a parallel study of working 
alliance in this setting. If this is the case there may be a need to develop interventions that can be delivered over one to three sessions, and to focus the sessions on what Kenyan patients find most useful. It may also be important to engage in task-shifting models to equip primary healthcare providers to deliver basic counselling or psychological first aid, in order to minimise the need for patients to travel long distances to get treatment.

\section{Conclusions}

When trying to improve the effectiveness of psychological therapies in non-Western cultures, there may be a risk of 'empirical imperialism' - that is, imposing on local practitioners theories and methods developed in Western cultures despite lack of culture-specific evidence of effectiveness. We set out an alternative strategy that utilises initial effectiveness data as information on which patient groups need improved treatments and on what practices are effective. With limited resources, we want time and money to be utilised effectively to develop evidence-based contextualised therapies, which can be delivered by the available staff in the minimum effective number of sessions.

\section{References}

Chowdhary, N., Sikander, S., Atif, N., et al (2014) The content and delivery of psychological interventions for perinatal depression by non-specialist health workers in low and middle income countries: $a$ systematic review. Clinical Obstetrics and Gynaecology, 28, 113-133.

Falkenström, F. (2010) Does psychotherapy for young adults in routine practice show similar results as therapy in randomized clinical trials? Psychotherapy Research, 20, 181-192.
Gisore, P., Kaseje, D., Were, F., et al (2014) Motivational interview ing intervention on health-seeking behaviors of pregnant women in western Kenya. Journal of Applied Biobehavioral Research, 19 $144-156$

Holmqvist, R., Philips, B. \& Barkham, M. (2015) Developing practice-based evidence: benefits, challenges, and tensions. Psychotherapy Research, 25, 20-31.

Issa, B. A., Yussuf, A. D., Abiodun, O. A., et al (2015) Hospital doctors' management of psychological problems at a Nigerian tertiary health institution. BJPsych International, 12, S11-S15.

Lampropoulos, G. K., Goldfried, M. R., Castonguay, L. G., et al (2002) What kind of research can we realistically expect from the practitioner? Journal of Clinical Psychology, 58, 1241-1264.

Möller-Leimkühler, A. M. (2002) Barriers to help-seeking by men: a review of sociocultural and clinical literature with particular reference to depression. Journal of Affective Disorders, 71, 1-9.

Muriungi, S. K. \& Ndetei, D. M. (2013) Effectiveness of psychoeducation on depression, hopelessness, suicidality, anxiety and substance use among basic diploma students at Kenya Medical Training College. South African Journal of Psychiatry, 19, 41-50.

Murray, L. K., Dorsey, S., Haroz, E., et al (2014) A common elements treatment approach for adult mental health problems in low- and middle-income countries. Cognitive and Behavioral Practice, 21, $111-123$

Papas, R. K., Sidle, J. E., Gakinya, B. N., et al (2011) Treatment outcomes of a stage 1 cognitive-behavioral trial to reduce alcohol use among human immunodeficiency virus-infected out-patients in Western Kenya. Addiction, 106, 2156-2166.

Roth, A. \& Fonagy, P. (2005) What Works for Whom: A Critical Review of Psychotherapy Research (2nd edn). Guilford Press.

Swift, J. K., Callahan, J. L., Ivanovic, M., et al (2013) Further examination of the psychotherapy preference effect: a metaregression analysis. Journal of Psychotherapy Integration, 23 $134-145$.

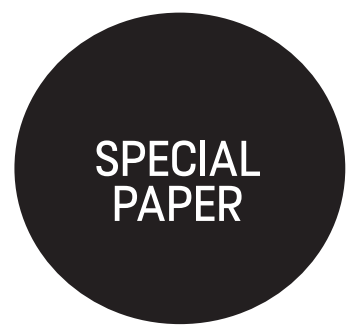

\title{
Edawu: a journey from in-patient rehabilitation to community-based treatment and rehabilitation in Nigeria
}

\author{
N. Chakraborty, ${ }^{1}$ A. Erinfolami ${ }^{2}$ and A. Lucas $^{3}$
}

Consultant Psychiatrist eicestershire Partnership NHS Trust, UK. email Nand Chakraborty@leicspart.nhs.uk ${ }^{2}$ Consultant Psychiatrist and Head of the Department of Psychiatry, University of Lagos, Nigeria

${ }^{3}$ Occupational Therapist, ACCEPT (mental health charity), UK

\begin{abstract}
Community-based rehabilitation is the strategy endorsed by the World Health Organization and other international bodies to promote the inclusion of people with disabilities, particularly in low- and middle-income countries. In this article we trace the journey of Edawu, a mental health rehabilitation unit in a rural area of Benue State, Nigeria, from an in-patient rehabilitation unit to a community-focused service. The partnership of organisations from the UK with Edawu along the journey is also described. The authors set out learning points from the project and the principles behind sustainable overseas organisational partnerships.
\end{abstract}

The WHO-AIMS report (WHO \& Ministry of Health, 2006) on mental health services in Nigeria states that information about the level of mental health services in Nigeria is difficult to assimilate. Systematic data gathering is non-existent. Government-funded services include stand-alone psychiatric hospitals and psychiatric units located in general hospital settings. Since the catchment areas are not strictly defined, it is impossible to determine the population for which the facilities cater.

This article concerns the Edawu community mental healthcare project, in a rural part of Benue State, in the south-west of Nigeria. Facilities for people who have a mental illness are sparse and reflect many of the findings from the WHO-AIMS 